\title{
Is the Optimal Load for Maximal Power Output During Hang Power Cleans Submaximal?
}

\author{
Seiichiro Takei, Kuniaki Hirayama, and Junichi Okada
}

\begin{abstract}
Purpose: The optimal load for maximal power output during hang power cleans (HPCs) from a mechanical perspective is the 1-repetition-maximum (1RM) load; however, previous research has reported otherwise. The present study thus aimed to investigate the underlying factors that determine optimal load during HPCs. Methods: Eight competitive Olympic weight lifters performed HPCs at 40\%, 60\%, 70\%, 80\%, 90\%, 95\%, and 100\% of their 1RM while the ground-reaction force and bar/body kinematics were simultaneously recorded. The success criterion during HPC was set above parallel squat at the receiving position. Results: Both peak power and relative peak power were maximized at 80\% 1RM (3975.7 [439.1] W, 50.4 [6.6] W/kg, respectively). Peak force, force at peak power, and relative values tended to increase with heavier loads $(P<.001)$, while peak system velocity and system velocity at peak power decreased significantly above $80 \% 1 \mathrm{RM}(P=.005$ and .011 , respectively). There were also significant decreases in peak bar velocity $(P<.001)$ and bar displacement $(P<.001)$ toward heavier loads. There was a strong positive correlation between peak bar velocity and bar displacement in 7 of 8 subjects $(r>.90, P<.01)$. The knee joint angle at the receiving position fell below the quarter-squat position above 70\% 1RM. Conclusions: Submaximal loads were indeed optimal for maximal power output for HPC when the success criterion was set above the parallel-squat position. However, when the success criterion was defined as the quarter-squat position, the optimal load became the 1RM load.
\end{abstract}

Keywords: Olympic weight lifting, power training, squat position, 1-repetition maximum

Olympic weight-lifting exercises are among the most powerful movements in sport and are often used by athletes for power enhancement along with other primary power exercises like plyometrics and jump squats. ${ }^{1}$ Among weight-lifting exercises, the hang power clean (HPC) is a widely used exercise in strength and conditioning settings. ${ }^{2,3}$ Many studies have shown the effectiveness of the use of optimal load to generate the highest power output. ${ }^{4,5}$ The reported optimal load for HPC varies among studies, ranging from $65 \%$ to $80 \%$ of 1 -repetition maximum (1RM). ${ }^{6-10}$ This inconsistency may derive from factors such as differences in the training stage of the subjects' yearly schedule and their strength levels. ${ }^{9,11}$

Olympic lifting is usually categorized as a ballistic exercise, along with bench press throws and jump squats. ${ }^{12,13}$ Ballistic exercise with an external load has little or no deceleration period, which results in the external load and/or the subject's body leaving the ground at the end of the concentric phase. ${ }^{12}$ From a mechanical point of view, the resulting vertical height of the external load and/or subject's body lifting off the ground is determined by the maximum vertical velocity during takeoff. According to the National Strength and Conditioning Association guidelines, in HPC, one should receive the bar in a quarter-squat position. ${ }^{14}$ Because of this narrow range of success criteria, the bar height lifted during HPC should be consistent for each lifter and independent of external load. In the relationship between height and velocity, velocity is not affected by varying loads. Because

(C) 2020 The Authors. Published by Human Kinetics, Inc. This is an Open Access article distributed under the terms of the Creative Commons Attribution 4.0 International License, CC BY 4.0, which permits unrestricted noncommercial and commercial use, distribution, and reproduction in any medium, provided the original work is properly cited, the new use includes a link to the license, and any changes are indicated. See http://creativecommons.org/licenses/by/4.0. This license does not cover any third-party material that may appear with permission in the article.

Takei is with the Graduate School of Sport Sciences, and Hirayama and Okada, the Faculty of Sport Sciences, Waseda University, Tokorozawa, Japan. Takei (seiichiro@fuji.waseda.jp) is corresponding author. the vertical ground reaction force during HPC increases as loads increase, $, 7,9,10,15$ and power is calculated as force multiplied by velocity, the power output during HPC should be maximized at 1RM.

However, previous studies have reported that submaximal loads are optimal for peak power output during HPC. ${ }^{6-10}$ There are 2 possible explanations for these results. First, the movement might not be executed correctly at heavy loads. Because it is not easy to perform ballistic exercises like HPC with correct technique and consistency at near-maximum loads, subjects must be proficient in HPC for quantification of the pure power characteristics of the movement. In previous studies, the research subjects were not Olympic weight lifters. Another possible factor for an optimal load below 1RM is the wide range of success criteria, compared with the quarter squat mentioned before, for the receiving position during HPC. In previous studies, the success criterion was the lifter's upper thigh above parallel to the ground ${ }^{6,7,9}$ or was not clearly described. ${ }^{8,10}$ In either case, the wide range of criteria could allow for a significant decrease in the height of the bar, and thus a decreased velocity at heavier loads. With the success criterion set at the quartersquat position as per the National Strength and Conditioning Association guidelines, the optimal load should be the 1RM.

Thus, the aim of the present study was to investigate the factors determining the optimum load for maximal power output during HPCs in Olympic weight lifters. We tested 2 hypotheses: (1) a decrease in velocity leads to a decrease in power output at loads above the optimal loads reported in previous studies $(65 \%-80 \%$ $1 \mathrm{RM})$, which is related to a significant decrease in lifted bar height and (2) 1RM is the optimal load when the success criterion is set at the quarter-squat position.

\section{Methods}

\section{Subjects}

Eight male weight lifters with at least 5 years of experience in Olympic lifting participated in this study. All of them participated 
in the national and/or international level competitions. Subject characteristics (mean [SD]) are as follows: age 21 (3) years, height 169.0 (4.2) cm, body mass 80.3 (14.9) kg, 1RM HPC 125.6 (14.5) $\mathrm{kg}$, and 1RM HPC 1.59 (0.17) kg/body mass. They often perform various power cleans in the catch position above the quarter squat besides cleans with a deeper catch position, especially during the preparatory phase of their yearly schedule. All testing sessions were conducted shortly after the preparatory phase. This study was approved by the ethical review board of Waseda University, and each subject provided informed consent. Because of our study design, the hypotheses were not disclosed until data collection was complete.

\section{Procedures}

Data collection took place over 2 days with the 1RM test of HPC and power test conducted on day 1 and day 2, respectively. HPC was performed as previously described. ${ }^{6-10,15}$ Briefly, (1) the subjects stood still on a force platform while holding the bar at their upper thigh, (2) moved the bar down to the upper part of their knees, (3) pulled the bar drastically upward with countermovement, and (4) then lowered themselves into a quarter-squat position to receive the bar on their shoulders. For 1RM HPC testing on day 1 , the subjects initiated the session with a warm-up of 2 sets of 5 repetitions of HPC with a 20-kg Olympic lifting bar. After finishing the warm-up sets, the subjects performed HPCs at $50 \%, 70 \%, 80 \%$, and $90 \%$ of their self-reported 1RM. The subjects then progressively increased the weight by 2 to $5 \mathrm{~kg}$ depending on their performance of the previous lift. The test was terminated when the subjects failed the lifts 2 times in a row. The success criterion was set at the subjects' upper thigh above parallel to the ground at the receiving position..$^{6,7,9}$ The power test (day 2 of testing) was conducted from 2 to 10 days after the $1 \mathrm{RM}$ test. The loads used in the power test were determined based on the subjects' 1RM obtained on day 1 of testing. As with the 1RM test, the session started after warm-up sets with a 20-kg Olympic lifting bar. HPCs were then performed at $40 \%, 60 \%, 70 \%$, and $80 \%$ 1RM with 2 attempts per load and 90\%, 95\%, and 100\% 1RM with 1 attempt per load on a force platform (0625, ACP, AccuPower; AMTI, Watertown, MA). The subjects were instructed to attempt each lift with their maximum effort. A rest period of 2 minutes was given between attempts at loads of $40 \%, 60 \%, 70 \%$, and $80 \%$ and 3 minutes at loads of $90 \%, 95 \%$, and $100 \%$ 1RM. As with the $1 \mathrm{RM}$ test, loads were gradually increased from $40 \%$ to $100 \% 1 \mathrm{RM}$ for injury prevention. Subjects were allowed to use tape, hook grips, knee sleeves, and weight belts during the 1RM and power tests.

\section{Measurement}

During the power test, all attempts were recorded from a sagittal plane at $120 \mathrm{~Hz}$ with a digital camera (15373667; FLIR Integrated Imaging Solutions Inc, British Columbia, Canada). Ana$\log$ data obtained from the force platform were digitally converted at a sampling rate of $1000 \mathrm{~Hz}$ using an analog-to-digital converter (EIRBZ22002369; CONTEC Co Ltd, Osaka, Japan) and then recorded in a personal computer. Force platform and video data were synchronized using Kinema Tracer analysis software (KISSEI COMTEC Co Ltd, Nagano, Japan). The vertical ground reaction force obtained from the force platform was used to determine the vertical acceleration and vertical velocity of the system (lifter's body mass plus external load) using forward dynamics. System power was calculated as the force value multiplied by the system velocity at each time interval (power $=$ force $\times$ velocity). Because the present study investigated power output during the second pull phase of HPC, only the propulsion phase was analyzed, that is, the phase from the instance when the system velocity changed from negative to positive, to the instance when the force fell $10 \mathrm{~N}$ below the system mass. The peak force, peak velocity, and peak power values were the highest during the propulsion phase of the force-time, velocity-time, and power-time data, respectively. The force at peak power and velocity at peak power were the values when peak power was expressed. To obtain those relative values, we divided the peak power, peak force, and force at peak power by each subject's body mass. The test-retest reliability of the current measuring protocol was previously validated by Suchomel et $\mathrm{al}^{6}$ with intraclass correlation coefficients ranging from .84 to .99. Twodimensional video analysis was performed to determine the bar kinematics data and knee joint angle during the receiving phase of HPC. Bar displacement data were filtered with a fourth Butterworth filter with a cutoff frequency of $6 \mathrm{~Hz}$. The displacement data were then converted to the vertical acceleration and velocity of the bar using inverse dynamics. The peak bar velocity was the highest value of the vertical velocity data, and the bar displacement was the vertical displacement from the bar height at peak bar velocity to the greatest bar height. The knee joint angle at the receiving position was the smallest angle formed by 2-dimensional position data of the greater trochanter, knee joint, and lateral malleolus. The position data for each joint were manually plotted.

\section{Statistical Analyses}

All results are expressed as the mean (SD). Normality of data was analyzed using the Shapiro-Wilk test. One-way repeated-measures analysis of variance (ANOVA) was used to compare each load's peak power, relative peak power, peak force, relative peak force, force at peak power, relative force at peak power, peak system velocity, system velocity at peak power, peak bar velocity, bar displacement, and knee joint angle at the receiving position. When significant differences were found, post hoc tests with Bonferroni correction were used for paired comparisons. Partial eta squared $\left(\eta_{\mathrm{p}}^{2}\right)$ was also calculated to indicate the effect size in ANOVA tests. Cohen $d$ effect sizes between the optimal load and other loads were calculated to display practical significance. Effect sizes of 0.00 to $0.19,0.20$ to $0.59,0.60$ to $1.19,1.20$ to $1.99,2.00$ to 3.99 , and $\geq 4.00$ were interpreted as trivial, small, moderate, large, very large, and nearly perfect, respectively. ${ }^{16}$ For loads with data for 2 attempts, the one that generated the higher peak power was used for analysis. Pearson correlation coefficient $(r)$ was used to assess relationships between bar displacement and peak bar velocity for each subject. The significance level was set at $P<.05$. All statistical analyses were performed using SPSS version 23 (IBM Corp, New York, NY).

\section{Results}

\section{System Power, Force, and Velocity}

The ANOVA results indicated that relative intensity had significant effects on peak power $\left(P=.006, \eta_{\mathrm{p}}^{2}=.595\right)$, relative peak power $\left(P=.002, \eta_{\mathrm{p}}^{2}=.644\right)$, peak force $\left(P<.001, \eta_{\mathrm{p}}^{2}=.774\right)$, relative peak force $\left(P<.001, \eta_{\mathrm{p}}^{2}=.838\right)$, force at peak power $(P<.001$, $\left.\eta_{\mathrm{p}}^{2}=.808\right)$, relative force at peak power $\left(P<.001, \eta_{\mathrm{p}}^{2}=.859\right)$, peak system velocity $\left(P=.005, \eta_{\mathrm{p}}^{2}=.572\right)$, and system velocity at peak power $\left(P=.011, \eta_{\mathrm{p}}^{2}=.473\right)$. The post hoc test results and Cohen $d$ values are presented in Table 1 . 


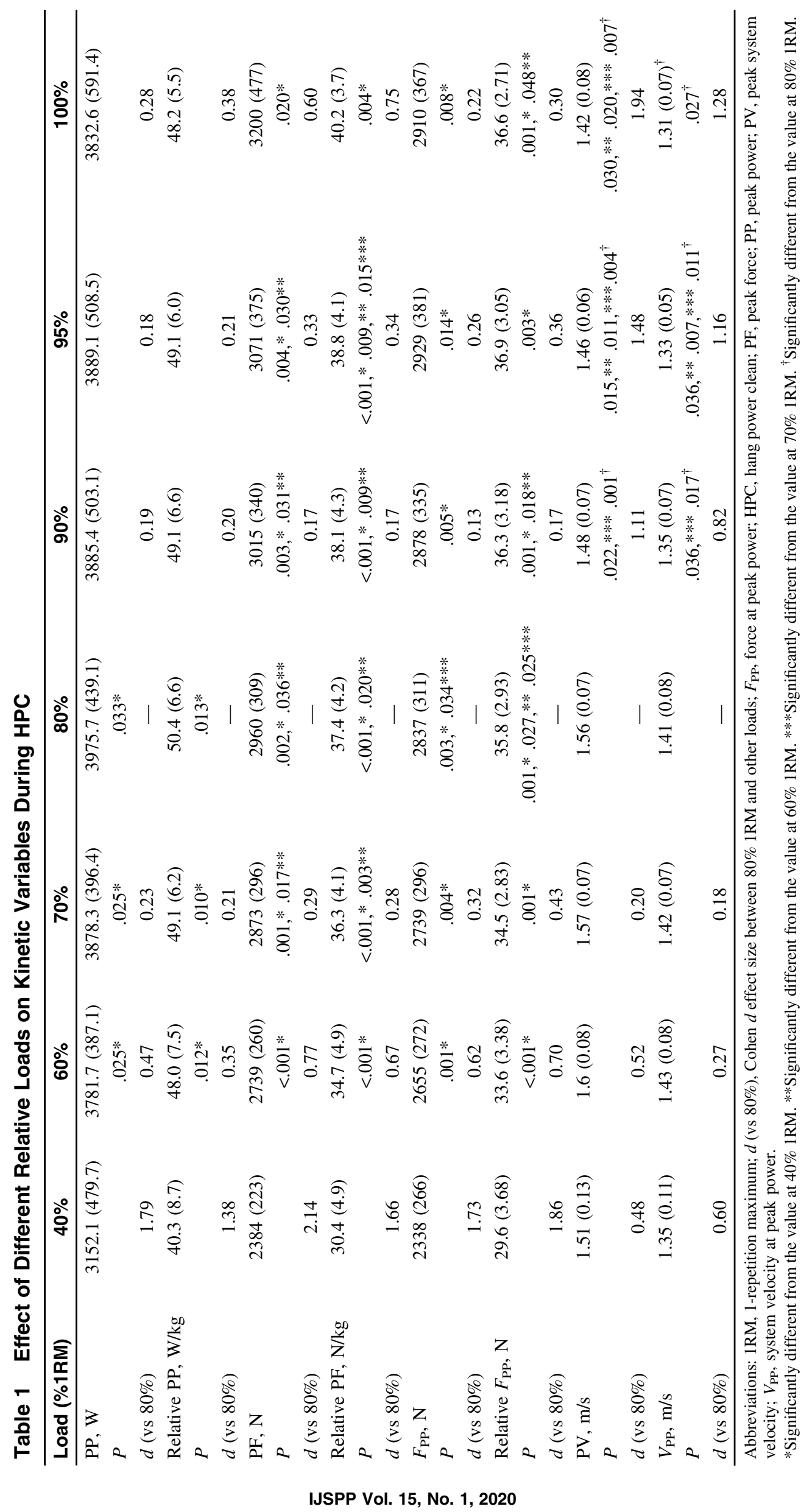


Both peak power and relative peak power at $60 \%, 70 \%$, and $80 \% 1 \mathrm{RM}$ were significantly higher than peak power at $40 \%$ $1 \mathrm{RM}$, and both were maximized at $80 \% 1 \mathrm{RM}$. Peak force and relative peak force at $60 \%, 70 \%, 80 \%, 90 \%, 95 \%$, and $100 \% 1 \mathrm{RM}$ were significantly higher than $40 \% 1 \mathrm{RM}$, and peak force and relative peak force at $70 \%, 80 \%, 90 \%$, and $95 \% 1 \mathrm{RM}$ were significantly higher than $60 \% 1 \mathrm{RM}$. Relative peak force at $95 \%$ 1RM was significantly higher than 70\% 1RM. In addition, both force at peak power and relative force at peak power at $60 \%, 70 \%$, $80 \%, 90 \%, 95 \%$, and $100 \% 1 \mathrm{RM}$ were significantly higher than at $40 \% 1 \mathrm{RM}$, and force at peak power and relative force at peak power at $80 \% 1 \mathrm{RM}$ were significantly higher than at $70 \% 1 \mathrm{RM}$. Relative force at peak power at $80 \%, 90 \%$, and $100 \% 1 \mathrm{RM}$ was significantly higher than at $60 \% 1 \mathrm{RM}$. Post hoc tests also revealed that peak system velocity at $95 \%$ and $100 \% 1 \mathrm{RM}$ was significantly lower than that at $60 \% 1 \mathrm{RM}$. In addition, peak system velocity at $90 \%, 95 \%$, and $100 \% 1 \mathrm{RM}$ was significantly lower than that at $70 \%$ and $80 \%$ 1RM. Similarly, system velocity at peak power at $95 \% 1 \mathrm{RM}$ was significantly lower than that at $60 \% 1 \mathrm{RM}$; system velocity at peak power at $90 \%$ and $95 \% 1 \mathrm{RM}$ was significantly lower than that at $70 \% 1 \mathrm{RM}$; and system velocity at peak power at $90 \%, 95 \%$, and $100 \% 1 \mathrm{RM}$ was significantly lower than that at $80 \% 1 \mathrm{RM}$.

\section{Bar Velocity and Displacement}

Analysis of variance confirmed the significant effect of relative load on peak bar velocity $\left(P<.001, \eta_{\mathrm{p}}^{2}=.870\right)$ as well as bar displacement $\left(P<.001, \eta_{\mathrm{p}}^{2}=.750 ;\right.$ Figure 1$)$. Peak bar velocity significantly decreased as load increased, with the exceptions of $60 \%$ versus $70 \%$ $1 \mathrm{RM}, 90 \%$ versus $95 \% 1 \mathrm{RM}$, and 95\% versus 100\% 1RM (all $P<.05)$. The peak bar velocity effect sizes of each load against $80 \%$ $1 \mathrm{RM}$ were $2.59,1.42,0.98,1.01,1.26$, and 1.49 for $40 \%, 60 \%$, 70\%, 90\%, 95\%, and 100\% 1RM, respectively. Regarding bar displacement, the values at $90 \%$ and $100 \% 1 \mathrm{RM}$ were significantly lower than the value at $40 \% 1 \mathrm{RM}(P=.042$ and .028 , respectively $)$,

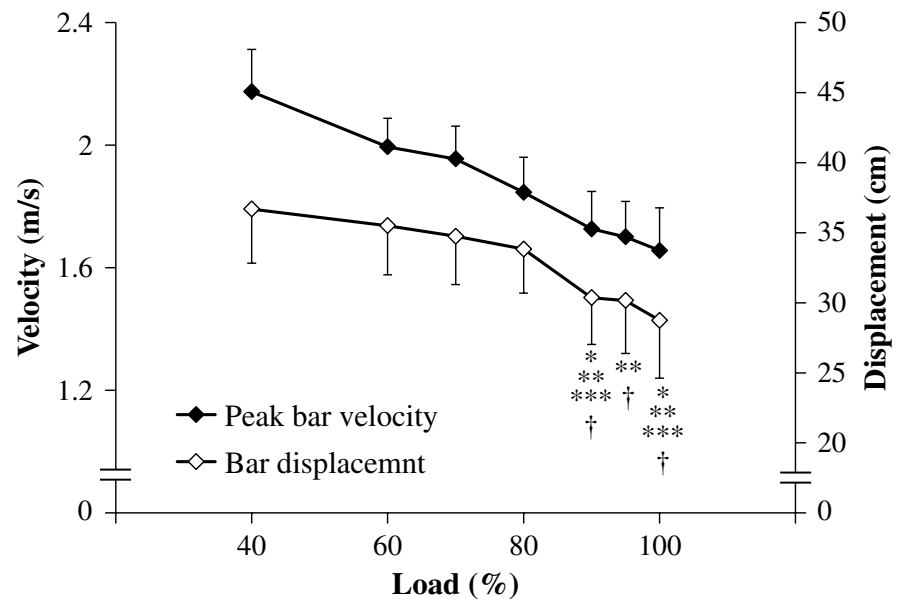

$\overline{\text { Figure } 1 \text { - Peak bar velocity and bar displacement at different relative }}$ loads during HPC. All values for peak bar velocity differ significantly from each other except for $60 \%$ versus $70 \%$ 1RM, 90\% versus $95 \%$ $1 \mathrm{RM}$, and $95 \%$ versus $100 \%$ 1RM. HPC indicates hang power clean; 1RM, 1-repetition maximum. * Significantly different from the value at $40 \% 1 \mathrm{RM}$. $* *$ Significantly different from the value at $60 \% 1 \mathrm{RM}$. ***Significantly different from the value at $70 \% 1 \mathrm{RM}$. ${ }^{\dagger}$ Significantly different from the value at $80 \% 1 \mathrm{RM}$. and the values at $90 \%, 95 \%$, and $100 \% 1 \mathrm{RM}$ were significantly lower than the value at 60\% 1RM $(P=.031, .042$, and .023 , respectively). Furthermore, the values at $90 \%$ and $100 \% 1 \mathrm{RM}$ were significantly lower than the value at $70 \% 1 \mathrm{RM}(P=.015$ and .009 , respectively), and the values at $90 \%, 95 \%$, and $100 \%$ $1 \mathrm{RM}$ were all significantly lower than the value at $80 \% 1 \mathrm{RM}$ $(P=.017, .029$, and .004 , respectively). The bar displacement effect sizes of each load against 80\% 1RM were 0.81, 0.50, 0.28, 1.07, 1.06 , and 1.39 for $40 \%, 60 \%, 70 \%, 90 \%, 95 \%$, and $100 \% 1 \mathrm{RM}$, respectively. Figure $2 \mathrm{~A}$ shows the strong correlations between peak bar velocity and bar displacement in all subjects $(r>.90)$ except subject $h$. Figure 2B depicts the peak power at different relative intensities for subject $\mathrm{h}$, who showed no such correlation and yielded the maximum peak power value at 100\% 1RM.

\section{Knee Joint Angle}

Analysis of variance revealed significant effects of external load on knee joint angle at the receiving position $\left(P<.001, \eta_{\mathrm{p}}^{2}=.893\right.$; Figure 3). In the post hoc test, the values at 70\%, 80\%, 90\%, 95\%, and $100 \% 1 \mathrm{RM}$ were significantly lower than the value at $40 \%$ $1 \mathrm{RM}(P=.032, .002,<.001,<.001$, and .001 , respectively $)$, and the values at $80 \%, 90 \%, 95 \%$, and $100 \% 1 \mathrm{RM}$ were significantly lower than the value at $60 \% 1 \mathrm{RM}(P=.001, .002,<.001$, and .003 , respectively). In addition, the values at $90 \%, 95 \%$, and $100 \% 1 \mathrm{RM}$ were significantly lower than the value at 70\% 1RM $(P=.004$, .001 , and .003 , respectively), and the values at $90 \%, 95 \%$, and $100 \% 1 \mathrm{RM}$ were significantly lower than the value at $80 \% 1 \mathrm{RM}$ $(P=.028,<.001$, and .029 , respectively). The knee joint angle effect sizes of each load against 80\% 1RM were 5.78, 3.48, 1.73, $1.54,2.12$, and 2.03 for $40 \%, 60 \%, 70 \%, 90 \%, 95 \%$, and $100 \%$ $1 \mathrm{RM}$, respectively. The bar was received at a position considered to be a quarter squat at $40 \%, 60 \%$, and $70 \% 1 \mathrm{RM}$ and at a half-squat position at $80 \%, 90 \%, 95 \%$, and $100 \% 1 \mathrm{RM}$.

\section{Discussion}

The present study examined the effects of relative load on peak power output during HPCs by Olympic weight lifters to elucidate the underlying factors that make submaximal loads optimal for peak power output. Peak power was maximized at 80\% 1RM when the success criterion was set above the parallel-squat position, which resulted from the significant decrease in velocity and bar displacement at loads $\geq 90 \%$ 1RM. Conversely, peak power was maximized at the 1RM load when the success criterion was set at the quarter-squat position. These results support our hypotheses, indicating that the success criterion is likely to determine the optimal load for HPC.

Both peak power and relative peak power were maximized at $80 \% 1$ RM during HPC, consistent with reports by Suchomel et $\mathrm{al}^{6}$ and Kilduff et al. ${ }^{10}$ However, because of the small sample size, the value at the optimal load was not statistically significant compared with most of the other loads, as previously reported.6,9,10 Cohen $d$ effect size revealed that the farther the compared load from $80 \% 1 \mathrm{RM}$, the larger its practical significance (Table 1). Power was calculated as force multiplied by velocity. Because peak force and force at peak power tended to increase with heavier loads, it can be concluded that the decrease in peak power at loads $90 \%$ 1RM or heavier was caused by the significant decrease in peak system velocity and system velocity at peak power (Table 1). Bar displacement also decreased significantly for loads equal to $90 \%$ $1 \mathrm{RM}$ or heavier. These results supported our first hypothesis that 

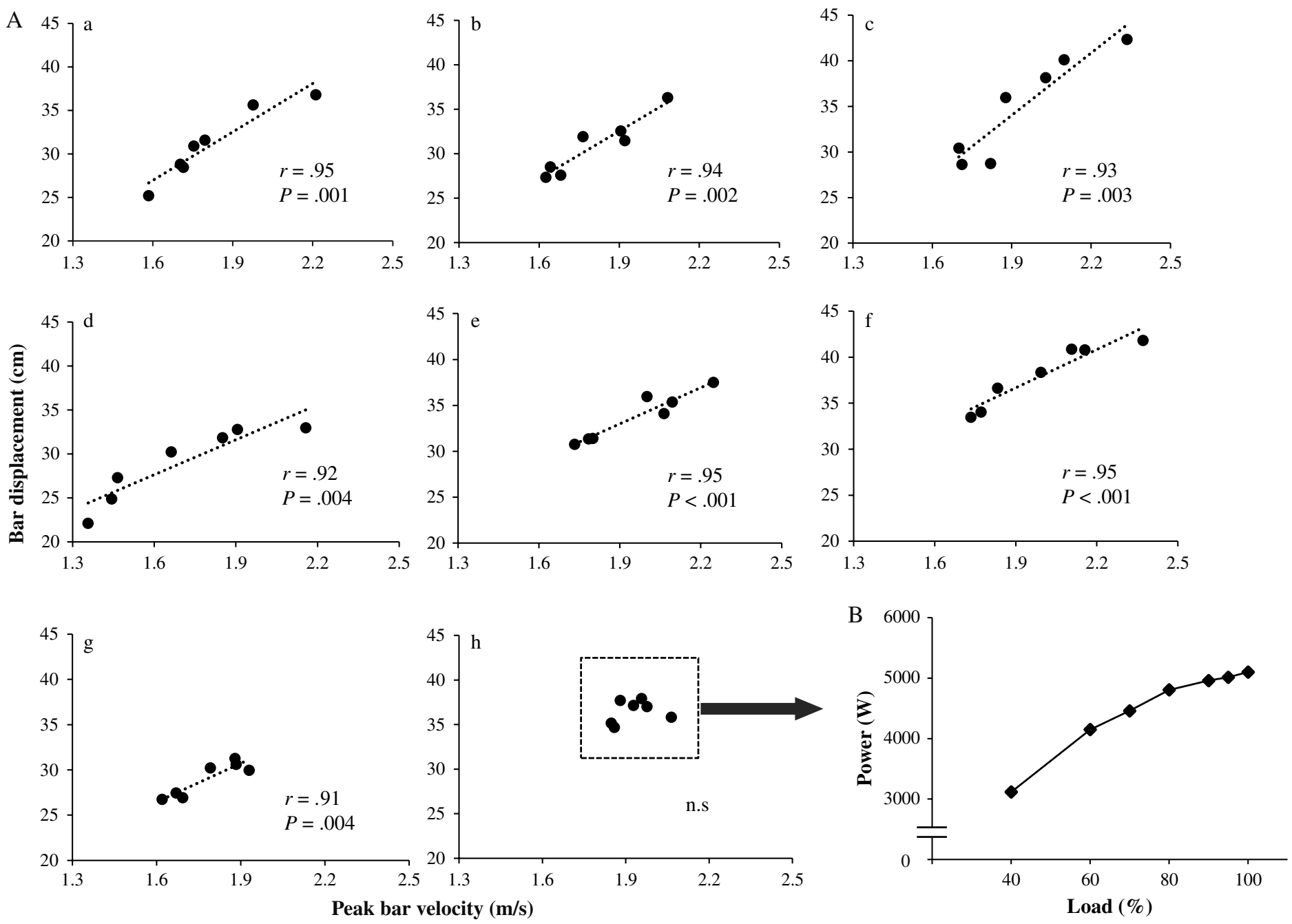

Figure 2 - (A) Scatterplots showing the correlation between peak bar velocity and bar displacement. Subpanels (a) to (h) present the correlation for each subject; $r$ is the correlation coefficient; n.s. means not significant. (B) Peak power at different relative intensities for subject h, who showed no correlation between peak bar velocity and bar displacement.

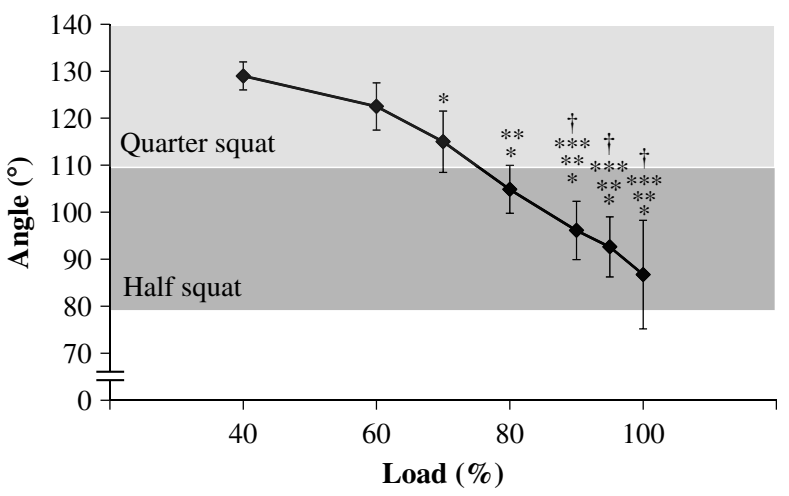

Figure 3 - Knee joint angle at the receiving position. The highlighted gray area shows the knee joint angle range of $110^{\circ}$ to $140^{\circ}$, which is considered a quarter-squat position. ${ }^{17,18} *$ Significantly different from the value at $40 \% 1 \mathrm{RM} . * *$ Significantly different from the value at $60 \% 1 \mathrm{RM}$. $* * *$ Significantly different from the value at $70 \% 1 \mathrm{RM}$. ${ }^{\dagger}$ Significantly different from the value at $80 \%$ 1RM. 1RM indicates 1-repetition maximum. at loads heavier than the optimal loads reported in previous studies $(65 \%-80 \%$ 1RM), peak power would decrease significantly due to decreases in velocity relative to decreases in bar displacement.

When the criterion for a quarter squat was set at a knee joint angle of $110^{\circ}$ to $140^{\circ}$, consistent with the criterion used by Hartmann et $\mathrm{al}^{17}$ and Schoenfeld, ${ }^{18}$ loads where the subjects received the bar at the quarter-squat position or higher were $40 \%$ to $70 \% 1 \mathrm{RM}$ (Figure 3). In the present study, peak power increased with increment in relative load from $40 \%$ to $80 \%$ 1RM. If the success criterion was set exactly to the quarter-squat position, 1RM became the optimal load for HPC, which is consistent with our second hypothesis.

We adopted the same success criterion used in previous studies $^{6,7,9}$ such that HPC attempts were considered unsuccessful if the subject's upper thigh went below parallel to the ground at the receiving position. The results showed that the highest peak power was generated at a submaximal load of $80 \% 1 \mathrm{RM}$, which is consistent with optimal loads of $65 \%$ to $80 \% 1 \mathrm{RM}$ reported in the previous studies. ${ }^{6-10}$ The highest peak power was generated at a submaximal load due to the significant decrease in system velocity 
and bar velocity relative to the decrease in bar displacement (Table 1; Figure 1). During ballistic exercises like Olympic weight lifting, the velocity at the takeoff phase determines the resulting heights of the barbell and/or lifter (ignoring the trivial contribution of the upper extremities). Indeed, Figure 2A indicates strong positive correlations between peak bar velocity and bar displacement in 7 of 8 subjects. The success criterion used in the previous studies allowed the bar height to decrease significantly at heavier loads. This coincided with a decrease in system velocity and bar velocity, resulting in a decrease in peak power at $90 \% 1 \mathrm{RM}$ or greater (Table 1; Figure 1). Taking this into consideration, the reason for the optimal load during HPC being submaximal may be because the success criterion was set above a parallel squat, which is relatively wide. The only subject who did not show any salient change in bar velocity or displacement across the different relative loads (subject h) generated the highest peak power at 100\% 1RM, which supports our point that the optimal load is 1RM when a narrower range of criterion is adopted, namely the quarter-squat position.

The optimal load differed on the basis of whether the success criterion adopted was narrow as a quarter-squat position or wide as a parallel-squat position as in the previous studies. The results in Figure 3 suggest that with a narrow criterion, even though the load that maximizes peak power output does not change, the optimal load becomes $100 \%$ 1RM. This implies that if the receiving position was consistent regardless of relative load, then the optimal load would always be the maximal load; in this regard, there should not be any differences among individuals or studies. In previous works, it was suggested that inconsistencies in optimal load were due to differences in the subjects' strength, fatigue, and training stage..$^{9,11}$ However, based on our findings (Figure 3), the lifters' receiving positions might not have been strictly controlled, which could be the primary factor for such inconsistency. It has been stated in a journal article, ${ }^{19}$ a review article, ${ }^{1}$ and original research articles ${ }^{6-10}$ that the optimal load for Olympic weight-lifting exercises that include the motion of receiving the bar after the second pull phase is a submaximal load. However, because it is possible that the success criterion (quarter squat vs parallel squat) is a determinant of the optimal load for HPC, the results of the previous studies and their interpretations should be applied with caution in strength and conditioning settings.

\section{Practical Application}

Consistent with previous studies, peak power during HPCs was seemingly maximized at $80 \% 1 \mathrm{RM}$ in the present study. However, this finding was limited to cases where the success criterion for the receiving position was set above the parallel-squat position. By contrast, in the standard quarter-squat position, the optimal load was the maximal load. Thus, when using HPCs for power training, coaches and athletes should select the appropriate \%1RM load based on the desired receiving position.

\section{Conclusions}

When the success criterion is set above the parallel-squat position, a submaximal load is indeed the optimal load for maximal power output in HPCs. The decreased peak power at loads $\geq 90 \%$ derives from the significant decrease in velocity and bar displacement. However, when the success criterion is set exactly to the quartersquat position, 1RM becomes the optimal load.

\section{Acknowledgments}

The authors would like to thank the weight-lifting team members of Waseda University for their participation in the study. They also wish to acknowledge the help provided by Mr Yoshiki Morikawa, Mr Hisato Orai, and Ms Saaya Uchikado for their assistance with the data collection. This work was supported by JSPS KAKENHI Grant Number JP17K01696 and Waseda University Grant for Special Research Projects (project number: 2017K-322). The results of the current study do not constitute endorsement of the product by the authors or the journal.

\section{References}

1. Cormie P, McGuigan MR, Newton RU. Developing maximal neuromuscular power: part 2-training considerations for improving maximal power production. Sports Med. 2011;41:125-146. PubMed ID: 21244105 doi:10.2165/11538500-000000000-00000

2. Ronai P, Scibek E. The hang power clean. ACSMs Health Fit J. 2016;20:50-55.

3. Hori N, Newton RU, Andrews WA, Kawamori N, McGuigan MR, Nosaka K. Does performance of hang power clean differentiate performance of jumping, sprinting, and changing of direction? J Strength Cond Res. 2008;22:412-418. PubMed ID: 18550955 doi:10.1519/JSC.0b013e318166052b

4. Kaneko M, Fuchimoto T, Toji H, Suei K. Training effect of different loads on the force-velocity relationship and mechanical power output in human muscle. Scand J Med Sci Sports. 1983;5: $50-55$.

5. Wilson GJ, Newton RU, Murphy AJ, Humphries BJ. The optimal training load for the development of dynamic athletic performance. Med Sci Sports Exerc. 1993;25:1279-1286. PubMed ID: 8289617 doi:10.1249/00005768-199311000-00013

6. Suchomel TJ, Beckham GK, Wright GA. The impact of load on lower body performance variables during the hang power clean. Sports Biomech. 2014;13:87-95. doi:10.1080/14763141.2013. 861012

7. Suchomel TJ, Wright GA, Kernozek TW, Kline DE. Kinetic comparison of the power development between power clean variations. J Strength Cond Res. 2014;28:350-360. PubMed ID: 23689341 doi:10.1519/JSC.0b013e31829a36a3

8. Suchomel TJ, Sole CJ. Power-time curve comparison between weightlifting derivatives. J Sports Sci Med. 2017;16(3):407-413. PubMed ID: 28912659

9. Kawamori N, Crum AJ, Blumert PA, et al. Influence of different relative intensities on power output during the hang power clean: identification of the optimal load. J Strength Cond Res. 2005;19:698708. PubMed ID: 16095428

10. Kilduff LP, Bevan H, Owen N, et al. Optimal loading for peak power output during the hang power clean in professional rugby players. Int J Sports Physiol Perform. 2007;2:260-269. PubMed ID: 19168926 doi:10.1123/ijspp.2.3.260

11. Stone MH, O’Bryant HS, McCoy L, Coglianese R, Lehmkuhl M, Schilling B. Power and maximum strength relationships during performance of dynamic and static weighted jumps. J Strength Cond Res. 2003;17:140-147. PubMed ID: 12580669

12. Maloney SJ, Turner AN, Fletcher IM. Ballistic exercise as a preactivation stimulus: a review of the literature and practical applications. Sports Med. 2014;44:1347-1359. PubMed ID: 24943044 doi:10.1007/s40279-014-0214-6

13. Suchomel TJ, Lamont HS, Moir GL. Understanding vertical jump potentiation: a deterministic model. Sports Med. 2016;46:809-828. PubMed ID: 26712510 doi:10.1007/s40279-015-0466-9 
14. Caulfield S, Berninger D. Exercise technique for free weight and machine training. In: Haff GG, Triplett NT, eds. Essentials of Strength Training and Conditioning. 4th ed. Champaign, IL: Human Kinetics; 2015:351-408.

15. Suchomel TJ, Sole CJ. Force-time-curve comparison between weight-lifting derivatives. Int J Sports Physiol Perform. 2017;12(4): 431-439. doi:10.1123/ijspp.2016-0147

16. Hopkins WG. A scale of magnitude for effect statistics. https://www. sportsci.org/resource/stats/effectmag.html. Updated August 7, 2006. Accessed February 3, 2019.
17. Hartmann H, Wirth K, Klusemann M. Analysis of the load on the knee joint and vertebral column with changes in squatting depth and weight load. Sports Med. 2013;43:993-1008. PubMed ID: 23821469 doi:10.1007/s40279-013-0073-6

18. Schoenfeld BJ. Squatting kinematics and kinetics and their application to exercise performance. J Strength Cond Res. 2010;24:34973506. PubMed ID: 20182386 doi:10.1519/JSC.0b013e3181bac2d7

19. Suchomel TJ, Comfort P, Lake JP. Enhancing the force-velocity profile of athletes using weight-lifting derivatives. Strength Cond J. 2017;39:10-20. doi:10.1519/SSC.0000000000000275 